\title{
Malaria Control Strategies Adopted by Residents of Ikirike Community in Enugu State
}

\author{
Authors \\ Ishaku Salamatu ${ }^{1}$, Onwe, Simon Nwigboji ${ }^{2}$ and Gaji, Luka Dung ${ }^{3}$ \\ ${ }^{1}$ College of Nursing and Midwifery, Kafanchan \\ ${ }^{2}$ Department of Nursing, Ebonyi State University, Abakaliki \\ ${ }^{3}$ Department of Nursing, University of Jos, Plateau State \\ Corresponding Author \\ Ishaku Salamatu \\ Address: College of Nursing and Midwifery, Kafanchan \\ Phone: +2347037079252, +2348173614343 \\ Email: salamishaku0507@gmail.com
}

\begin{abstract}
Malaria is highly endemic in Nigeria; it poses a major challenge to the country as it impedes human development. The economic loss to Nigeria due to malaria is estimated at 132 billion naira annually due to loss of man hours resulting from sickness absence and cost of treatment. The study aims include Identifying the measures used to prevent and treat malaria by residents of Ikirike, finding out how the residents of Ikirike utilize the measures to prevent and treat malaria and assessing the perception of the residents of Ikirike on the effectiveness of the measures they use to prevent and treat malaria. Descriptive cross-sectional survey was used in this study. The findings from the study revealed that the malaria control strategies used by most of the respondents was drug, followed by barrier/drugs, barrier and environmental care in that order. Majority of the respondents do not have ITN. Out of 48\% who have ITN, $16.7 \%$ were not using it. The malaria control strategy that is most effective is drug; environment had the next highest frequency followed by barrier method in that order. Few respondents use insecticide spray while doors and windows are closed.
\end{abstract}

\section{Introduction}

Malaria is an infectious disease caused by parasite of the genius plasmodium. The four identified species of this parasite causing human malaria are plasmodium falciparum, plasmodium vivax, plasmodium ovale, and plasmodium malariae. These species of mosquito breed in clear stagnant water especially in unused discarded tyre, broken pots and other area where water can collect (Lagos
State MOH, 2015). In Nigeria, ninety eight percent $(98 \%)$ of all cases of malaria is due to plasmodium falciparum. This is the specie that is responsible for the severe form of the disease that leads to death. It is transmitted from bites of infected female anopheles mosquitoes to man (Lagos State MOH, 2015).

Malaria is highly endemic in Nigeria; it poses a major challenge to the country as it impedes 
human development. It is both a cause and consequence of underdevelopment and remains one of the leading causes of morbidity and mortality in the country. The economic loss to Nigeria due to malaria is estimated at 132 billion naira annually due to loss of man hours resulting from sickness absence and cost of treatment. It is a major cause of absenteeism from work and school. It contributes to poverty and result in poor pregnancy outcome (Lagos State MOH, 2015).

The burden of the disease has been a major source of concern to Government and development partners. The expression of this challenge has been translated through many instruments such as Abuja Declaration in year 2000, Universal Access to HIV/AIDS, tuberculosis and malaria (ATM) in 2006 and Millennium Development Goals to reduce disease burden by 2015 (Lagos State MOH, 2015).

Malaria is a difficult disease to control largely due to the highly adaptable nature of the vector and parasite involved. While effective tools have been and will continue to be developed to combat malaria, inevitably, over time the parasites and mosquitoes will evolve means to circumvent those tools if used in isolation or used ineffectively. To achieve sustainable control over malaria, health professionals need a combination of new approaches and tools, and research will play a critical role in development of these next generation strategies (National Institute of Allergy and Infectious Diseases, 2011).

The mechanism for the National Malaria Control Programme in Nigeria are the use of drug; chemotherapy and chemoprophylaxis, barrier approach include the use of chemical agents and insecticide treated net, chemical control using insecticides, Insecticide Treated Net which include short and long acting, environmental management approach and integrated vector management which is built on selected vector control strategies.

\section{Pathophysiology of malaria}

All the species are introduced into the human blood stream through the bite of an infected anopheles mosquito, the life stage of malaria at this stage is called sporozoite, they pass first to the liver where they undergo an initial stage of replication (exo-erythrocytic replication) before passing back into the blood and invading the Red blood cells (erythrocytes) hence this is the erythrocytic stage of the cycle. The malaria parasites that invade RBCs are merozoites, within the cell they replicate again bursting out once they have completed a set number of divisions, it is this periodic rupturing of RBCs that causes most of the symptoms associated with malaria, as the host's immune system responds to the waste product of the malaria parasites, metabolites hemozoin (malaria pigment) and the debris from the destroyed RBCs.

Different species of malaria rupture the RBCs at different intervals which leads to cycle of fever that characterize malaria. In order to replace the destroyed RBCs, the activities of the reticuloendothelial system particularly in the liver and spleen increase which leads to their enlargement. Plasmodium falciparum produces unique pathological effects, when it invade RBCs it makes them sticky to the walls of tiny blood vessels deep within major organs especially the kidneys, lungs, heart, brain, gut and the placenta (sequestration) which result in reduced blood flow to these organs causing the severe clinical symptoms associated with this infection such as in cerebral malaria characterize by severe headache, drowsiness, confusion and ultimately coma (Clark \& Cowden, 2003).

\section{Malaria Control Strategies \\ Environmental Management Approach}

Several efforts made at improving the state of the Nigeria environment is diverse and varied. These include legislative initiatives, creation of relevant agencies and evolution of policy instruments. Federal government's intervention in environmental management include the 
establishment of the Federal Environmental Protection Agency (FEPA) by Decree 58 of 1988, the National Policy on Environment of 1989 as well as the creation of the Federal Ministry of Environment in 1999. Most states of the federation have enacted laws which established waste and environmental sanitation Authorities/Boards and Environmental Protection Agencies while local governments have passed by-laws and regulations on various aspects of environment management. In 2004, Federal Ministry of Environment passed into Law Environmental Sanitation Policy. However, past efforts have been piece meal, adhoc and uncoordinated. The failure of past efforts could be attributed to poor environmental sanitation education and awareness, low literacy levels, indiscipline and disregard for the rule of law.

Environmental management approaches essentially depend on the source reduction and elimination of breeding sites. Drainage clearing, filling burrow pits and abandoned ditches, holes, flushing, drying of breeding places, management of irrigation water, removing shade where mosquitoes breed disposal of empty cans, discarded tyres are some of the activities to offset the breeding places of mosquitoes. These activities will be implemented in semi/urban areas in collaboration with relevant authorities to complement other interventions (Federal Ministry of Health, 2012).

\section{Barrier Approach}

Barrier approach include the use of chemical agents and insecticide treated net (ITN).

Chemical Control using Insecticides: Chemical control may be too expensive for countries with slender resources, widespread application of insecticide is not recommended as a primary healthcare strategy for the control of malaria. Chemical control also becomes more difficult as many Anopheles species develop resistance to conventional insecticides.

Insecticide Treated Net (ITN): Insecticide treated bed nets have proved effective in reducing morbidity and mortality due to malaria in some parts of Africa, including Nigeria. ITN can reduce child and infant morbidity and mortality due to malaria, as well as reduce malaria in pregnancy with significant reduction in parasitaemia, risk of low birth weight, still birth, abortion, intra-uterine growth retardation and other complications of malaria in pregnancy.

ITNs repels, inhibit or kill mosquitoes this giving protection to individuals sleeping under them. The ITN in addition to repelling mosquitoes from biting also shortens their life span, thereby preventing them from transmitting their infection. The insecticides use must meet the WHO insecticide evaluation scheme in terms of safety, efficacy, ease of application and cost effectiveness.

Insecticides endorsed for use in Nigeria include: alpha-cypermethrin, delta-methrin, etofenpox, permethrin, cyfluthrin and lamda-cyhalothrin insecticides. The first three are currently registered with NAFDAC. Re-treatment of the bed nets should be carried out at six monthly intervals. The long lasting insecticide treated nets (LLITNs) retain their insecticidal effects for much longer period/years (Obionu, 2007).

\section{The use of drugs}

\section{Chemotherapy}

Early diagnosis and treatment for active malarias using chloroquine and amodiaquine have remained the most active drugs for a long time. Quinine is still of value in the treatment of chloroquine-resistant falciparum malaria, and for potential administration in very severe infections sulfadoxine/pyrimethamine (fansidar), sulfene/ pyrimethamine (metakelfin), halofantrine (Halfan) and mefloquine (larian) have been used for the treatment of chloroquine-resistant malaria, or when there is a history of severe chloroquine induced pruritus (Obionu, 2007).

Increasing problems of drug resistance (especially chloroquine resistance) have led to current recommendations for the use of artenisinin derivates (artesunate, artemether, artemotile, 
dihydroartemesinin). Also to counter the treatment of resistance of plasmodium falciparum to monotherapies and improve treatment outcome, combinations of antimalarias, in particular artemisinin-based combinations are now recommended by the WHO for the treatment of falciparum malaria (Obionu, 2007).

Some of the currently recommended artemisininbased combination treatments (ACTs) include:

Artemether - inmefantrine (co-artemether)

Artesunate + amodiaquine or chloroquine

Artesunate + mefloquine

Artesunate + sulfadoxine/pyrimethamine

Combination therapy for malaria has become popular as they not only enhance antimalarial effectiveness but also delay the emergence of resistance.

Chemoprophylaxis: This implies the use of antimalarial drug to prevent malaria before infection occurs or before it becomes obvious. The aim is to prevent occurrence of the disease by acting on the parasites while still confined to the liver (causal or true prophylaxis), or to prevent any of the disease symptoms by suppressing the number of malaria parasites in the blood to such low level that they will not cause any clinical symptoms (clinical prophylaxis or suppression). In highly endemic areas the use of chemoprophylaxis is limited to people who are at high risk from severe and complicated malaria, and these include pregnant women, persons with sickle cell disease and non-immune visitors or residents (Obionu, 2007).

The World Health Organization (WHO) recommends that pregnant women in malaria endemic areas should receive two doses of sulphadoxine-pyrimethamine given at therapeutic doses at scheduled interval during the index pregnancy (Lagos State Ministry of Health, 2012).

\section{Integrated Vector Management (IVM)}

Integrated vector management (IVM) as recommended by WHO/AFRO is the main control strategy in Nigeria. The concept of integrated vector management (IVM) is built on selected vector control strategies and it involves targeted use of different vector control methods alone or in combination to prevent or reduce vector populations and human-vector contact costeffectively and in a sustainable manner. This is certainly in consonance with the Role Back Malaria (RBM) multiple vector control strategies, in carrying out IVM efforts are made to apply environmentally sound intervention (Federal Ministry of Health, 2012).

In terms of inter-sectoral collaboration, line ministries such as agriculture, water resources, environment, information, works and housing, education, universities, research institutes are involved in the implementation of IVM in the country. The general objective of IVM is the reduction of vector-borne disease particularly, malaria morbidity, through the prevention, reduction and or interruption of disease transmission, via the utilization of multiple control measures in a compatible manner (Federal Ministry of Health, 2012).

Jegede et al, (2006) conducted a study on what women do to prevent and treat malaria in Ibadan. Both qualitative and quantitative data were collected among Bodija market women in Ibadan. Questionnaires were administered among 501 market women and 10 in-depth interviews were conducted also among market women but who were nursing children at the time of the study. Data showed that although majority of the respondents used window nets, medicine $(95.2 \%)$ and insecticide $(80.0 \%)$ were mostly used to prevent malaria. Use of ITN was low with only $21.2 \%$ used it for children and $0.6 \%$ for self. Less than half of the respondents $(41.9 \%)$ have heard about it. Although self-medication (38.4\%) was the main method of treatment, use of herbs and hospital was on equal proportion of $30.3 \%$ and $30.9 \%$ respectively. Spiritual methods were rarely used $(0.4 \%)$. There is no significant relationship between age and treatment pattern $(\mathrm{p}>0.05$

Oladokun, Oladokun and Adesina (2011) conducted a study on the Knowledge and utilization of malaria control measures by 
pregnant and newly delivered mothers in Ibadan, Nigeria using cross sectional survey design. The objective was to describe the malaria preventive measures utilized by these women in this environment. The sample size was 1330 pregnant women in 132 facilities within Ibadan metropolis over a period of one year from April 2008 to April 2009. Consecutive sampling technique was used. A list of the registered government and private hospitals within Ibadan metropolis was obtained from the state ministry of health which contained the 132 hospitals and maternity centres were sampled. An average of 10 consecutively consented pregnant and newly delivered mothers who were in the hospital and the maternity centres at the time of visit were recruited into the study. Data collected was entered into the Microsoft excel file and analyzed using frequency tables with the SPPS 16 software package. The findings showed that the mean age of the respondents was 29.7. The modes of prevention most commonly reported as being effective were the use of insecticides spray, window nets and ITN in the index pregnancy and $67.2 \%$ of the women had had a drug administered for prophylactic purposes in the index pregnancy. It was concluded that, this study demonstrated awareness but poor use of control measures and poor use of recommended agents for the IPT. Hence the need to urgently explore and address the factors militating against the use of these preventive measures.

Adeneye, Jegede, Mafe and Nwokocha (2007). Conducted a pilot study to evaluate malaria control strategies in Ogun State, Nigeria. It was a cross sectional study with a sample size of 68,34 registered women attending ANC clinic and 34 mothers of children $<5$ years old. Questionnaire was use to collect data. The result showed that, most of the respondents $(63.2 \%)$ of the 68 respondents interviewed new about ITN, however, $21.1 \%$ were using ITN. Reasons given by those who do not use it include; they do not know about it prior to interview $(43.3 \%)$, they had no money (41.5\%), they did not know where to get it (7.6\%). It was concluded that efforts need to be intensified to make adequate information and material relating to different malaria control strategies more available and accessible at the community level to achieve and sustain the roll back malaria goals. However, a large study is needed to provide more generalized findings.

Efunshile, Amoo, Akintunde, Ojelekan, Konig and Konig (2011). Use and effects of malaria control measures in pregnancy at land maternity hospital, Lagos. The study lasted over a period of 6 months between July and December 2009. The sample size of 400 pregnant women that consented to participate in the study during this period was interviewed for the study. Women who were sick of malaria or any other form of illness were excluded from the study. Those who have taken anti-malaria drugs within the last 4 weeks were also excluded. Questionnaires was used to collect data on the socio-demographic characteristics, ITN usage, IPT usage and pregnancy history. Data was analyzed using freely available Graphpad Instat 3.06. Non parametric chi-square test was performed to evaluate the relationship between presence and absence of malaria in relation to the observed characteristics. Two-sided p-values of $<0.05$ indicate statistical significance. The findings revealed that, the most common malaria preventive measures was the use of insecticide spray (32.8\%), followed by the use of mosquito coil (27.5\%), only $15.5 \%$ depend on the use of ITN as the major mosquito control measure.

This study examined the malaria control strategies adopted by the residents of Ikirike Community in Enugu State, Nigeria.

\section{Purpose of the study}

The purpose of the study is to determine the malaria control strategies adopted by residents of ikirike community in Enugu State. The specific objectives are to:

1. Identify the measures used to prevent and treat malaria by residents of Ikirike. 
2. Find out how the residents of Ikirike utilize the measures to prevent and treat malaria.

3. Assess the perception of the residents of Ikirike on the effectiveness of the measures they use to prevent and treat malaria.

\section{Research Method}

Descriptive cross-sectional survey was used in this study; this method involves observing and describing the behaviour of the respondents without influencing it in any way (Martyn, 2008). This study was carried out in Ikirike community in Enugu South Local Government area of Enugu State. The population of this study comprised of all the residents of Ikirike community in Enugu South Local Government area of Enugu State.

\section{Out of all the residents of Ikirike community, 100 families were selected for this study.}

All the adults living in Ikirike community, who are willing to participate in the study and are available at the time of study, were included. The researchers used the convenience/availability sampling technique to recruit 100 families consecutively in to the study who meet with the inclusion criteria. The recruitment continued daily throughout the week until the required sample size was reached.

The researchers administered questionnaire was used to source information from the respondents. The questionnaire was developed from literature search on malaria control strategies and the three stated objectives. The questionnaire was divided into sections. Section 'A' demographic data. Section ' $B$ ' malaria control strategies. Section ' $C$ ' how malaria control strategies are used and ' $D$ ' effectiveness of the malaria control strategies. The respondents were to respond based on their views on the issue. A total of 14 items were generated.

A pilot test was conducted on 5 families in Obiago community using split-half method. The data collected was analyzed using Cronbach's alpha which gave a reliability of 0.84 . This showed that the instrument was reliable. Ethical approval was obtained orally from the village head of Ikirike community, Enugu State. Informed consent was obtained from each participant prior to the administration of the instrument. They were assured that all information given will be treated confidentially.

With the approval of the village head of Ikirike community, Enugu State and the consent of the respondents, the researchers administered the questionnaire to each participant that meets the inclusion criteria in their homes. Data collection lasted for a period of two weeks. The data gathered were analyzed using descriptive statistics such as frequency, percentages, mean and standard deviation. All the analyses were done using SPSS version 20.0 computer software programme.

\section{Discussion of Findings}

Report on the findings were done under: discussion of the major findings, implications of the findings, limitations of the study, suggestions for further studies, summary of the study, conclusions and recommendations.

\section{Malaria Control Strategies}

In this study, the malaria control strategies used by most of the respondents was drug, followed by barrier/drugs, barrier and environmental care in that order. This is true in this study because most of the respondents have primary education as their highest educational attainment. Since the more one is educated, the more the person is likely to use basic malaria control strategies like environmental care and barrier methods. But in this study, environmental care has the lowest scores.

The findings of Jegede et al (2006) supported this findings that medicine (95\%) and insecticides $(80 \%)$ were mostly used to prevent malaria. However, the findings of Jegede et al (2006) is in contrast to this findings that, majority of the respondents used window nets to prevent malaria. The finding is also supported by Efunshile, Amoo, 
Akintunde, Ojelekan, Konig and Konig (2011) that, most common malaria preventive measures was the use of insecticide spray (32.8\%), followed by the use of mosquito coil $(27.5 \%)$, only $15.5 \%$ depend on the use of ITN as the major mosquito control measure.

\section{How Malaria Control Strategies are Used}

Majority of the respondents do not have ITN. Out of $48 \%$ who have ITN, $16.7 \%$ were not using it. This is because, some people in this community belief that ITN kill people because of the chemicals used in treating the net. This finding agreed with Jegede et al (2006) that, use of ITN was low with only $21.2 \%$. This is because the study demonstrated poor use of malaria control measures.

Adeneye, Jegede, Mafe and Nwokocha (2007) also supported this finding that, most $63.2 \%$ of the 68 respondents interviewed knew about ITN, however, $21.1 \%$ were using ITN. Reasons given by those who do not use it include; they do not know about it prior to interview(43.3\%), they had no money $(41.5 \%)$, they did not know where to get it $(7.6 \%)$. It was concluded that efforts need to be intensified to make adequate information and material relating to different malaria control strategies more available and accessible at the community level to achieve and sustain the roll back malaria goals. However, a large study is needed to provide more generalized findings.

In this study, majority of the respondents (79\%) used orthodox drug for the prevention and treatment of malaria. Most of them obtained the drug from chemist or health facility. This is because, they understand the use of drug as a malaria control strategy better than other strategies. This findings is in contrast to that of Jegede et al (2006) that, self medication was the main method of treatment. Use of herbs and hospital was on equal proportion.
The Effectiveness of the Malaria Control Strategies

The malaria control strategy that is most effective is drug. Environment had the next highest frequency followed by barrier method in that order. Few respondents use insecticide spray while doors and windows are closed. This is because most of the respondents used drug as their malaria control strategy, hence they may not know how effective other strategies are.

This finding is in contrast to that of Oladokun, Oladokun and Adesina (2011) that, the modes of prevention of malaria commonly reported as being effective were the use of insecticides spray, window nets and ITN. It was concluded that, the study demonstrated awareness but poor use of malaria control measures; hence the need to urgently explore and address the factors militating against the use of these preventive measures.

Implications of the findings to Nursing Practice

Poor environmental care in terms of bad status of the drainage system, not clearing bushes, presence of empty cans and poor use of insecticides by the respondents will help the nurse to know the perspective of care to be rendered to them towards better utilization of these strategies.

Good use of drug to prevent and treat malaria by the respondents will help to reduce complications associated with malaria. Moreover, less use of barrier method particularly the ITN will help the nurse to educate the people on the importance of using it and correct misconception about it thereby increasing it usage.

Good use of orthodox medication mostly from chemist and health facility will help to reduce complications, drug resistance and improve clinical outcome.

\section{Limitations of the study}

It was difficult for the researcher to convince some of the respondents to participate in the study. 


\section{Conclusions}

The average malaria control strategies used by majority of the respondents were drug, followed by barrier and drug, barrier alone, environmental care respectively. The way malaria control measures were used showed environment has the highest frequency, followed by barrier and lastly drug. The effectiveness of the malaria control measures was highest with drug, followed by environment, and less with barrier method.

\section{References}

1. Adeneye, A.K., Jegede, A.S., Mafe, M.A \& Nwokocha, E.E (2007). A pilot study to evaluate malaria control strategies in Ogun State, Nigeria.

2. Carrington A. (2001). Malaria: Its Human Impact, Challenges and Control Strategies in Nigeria.

3. Clark. J.A., and Cowden., W.B., (2003). Pathophysiology of plasmodium falciparum.

4. Efunshile, M., Amoo, A.O.J, Akintunde, G.B., Ojelekan, O.D., Konig W., \& Konig, B. (2011). Use and effects of malaria control measures in pregnancy in Lagos, Nigeria. Korean journal of parasitology: 49(4), 365-371.

5. Federal Ministry of Health (2012). National Malaria Control Programme, Nigeria. Available from nmcpnigeria.org/?=nmcbp.

6. Lagos State Ministry of Health (2012). MCP Centre of Health Excellence MCP.

7. Lagos State Ministry of Health (2015). Malaria control programme. Retrieved from www.http: //lagosstateministryofhealth.com/prog. Accessed on November 18th, 2015.

8. Martyn, S (2008). Descriptive Research Design. Retrieved from www.http://freelance-writereditor.com. Accessed on November $16^{\text {th }}, 2015$.
9. National Institute of Allergy and Infectious Disease (2011). Malaria prevention and control strategies, U.S. Retrieved from www.niaid.nih.gov/topics/malaria/research .November 18th, 2015.

10. Oladokun, A., Oladokun., R.E \& Adesina., O.A (2011). Knowledge and utilization of malaria control measures by pregnant and newly delivered mothers in Ibadan, Nigeria. Journal of Africa Health Sciences; 11 (4):573-577.

11. Obionu C.N. (2007). Primary Healthcare for Developing Countries, $2^{\text {nd }}$ Edition. Nigeria, Ezu Books, Ltd.

12. Sheeram P., Abraham C., (1995). The Health Belief Model, in predicting health behaviour. Buckingham, open university press. 